\title{
Neural networks for analysis of trabecular bone in osteoarthritis
}

1 Natalia A. Khovanova PhD*

Assistant professor, School of Engineering, University of Warwick, Coventry, UK

2 Torgyn Shaikhina BEng

PhD student, School of Engineering, University of Warwick,

Coventry, UK
$3 \quad$ Kajal K. Mallick PhD

Associate professor, Warwick Manufacturing Group, Coventry, UK
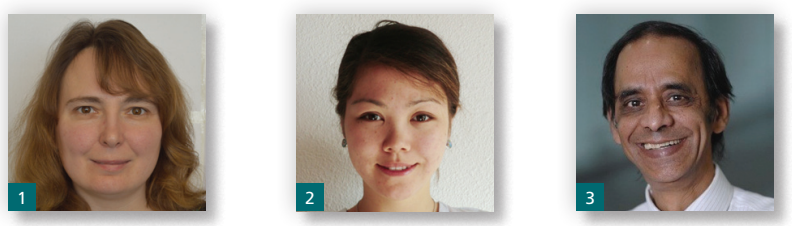

This study investigated the correlation of age in male and female specimens with physico-mechanical properties of trabecular bone including compressive strength, bone volume fraction, structural model index, trabecular thickness factor, level of inter-connectivity and pore morphology. An artificial neural network was designed to analyse 35 available samples in order to account for complex inter-dependencies of the key parameters in multi-dimensional space. Trained by using Levenberg-Marquardt back propagation algorithm, the network achieved regression factor of $\mathbf{0 . 9 6}$ by optimisation and showed that age correlates strongly with the physical properties of the bone affected by severe osteoarthritis. In addition, the compressive strength was found to be the most important factor for predicting the bone aging. Within the limitations of the input data set, the model developed provides a reliable predictive tool to tissue engineering applications.

\section{Introduction}

Bone is a natural biocomposite comprising hierarchical cortical and trabecular (cancellous) structures. The skeletal structurefunction relationship of trabecular tissue in vivo is complex ${ }^{1}$ since the mechano-biological properties are intrinsically dependent on the physical and geometrical parameters of bone, ${ }^{2}$ for example, three-dimensional (3D) trabecular architecture, ${ }^{3}$ pore size, compressive strength, ${ }^{4}$ yield strain and modulus, ${ }^{5,6}$ strain energy density and bone remodelling. The task of optimising or predicting these properties especially for trabecular bone becomes even more onerous due to the fact that mechanical properties of bone differ according to their anatomical location. ${ }^{7}$ Tissue engineers are thus often faced with problems of selecting the most successful strategy for both the design and fabrication of synthetic scaffold for the treatment of patients suffering from degenerative orthopaedic diseases triggered by osteoarthritis, osteoporosis, trauma, injury and metastatic cancer occurring in specific age group and gender. ${ }^{8,9}$ Computational techniques such as finite-element analysis (FEA) and other mathematical procedures have been used for clinical data in hip fractures with limited success. ${ }^{10-12}$ Phenomenological (data-driven) models, based on the experimental or clinical data, are known to have poor accuracy and restricted by the size of available data sets. ${ }^{13}$ Resulting statistical models are generally very sensitive to the inaccurate data and outliers, as well as the level of ambiguity allowed by the fuzzy logic and soft algorithms. This necessitates generation of large volume and high-quality data, and in tissue engineering, these requirements are highly impractical and often unrealistic. To resolve this dilemma and make statistical datadriven models applicable, it is desirable to design a model based on the principles of machine learning, which enables the ability of such model to learn from the additional data and to dynamically adapt to new inputs. ${ }^{14-17}$

Among various machine-learning data-driven algorithms, artificial neural network (ANN) is a powerful statistical tool of multidimensional data analysis genre. It enables modelling of multidimensional relationship of experimental data such as pattern recognition, clustering and function fitting. This avoids significant 
Bioinspired, Biomimetic and Nanobiomaterials

Volume 4 Issue BBN1
Neural networks for analysis of trabecular

bone in osteoarthritis

Khovanova, Shaikhina and Mallick complexity penalties of increased dimensionality typically associated with most statistical modelling. ${ }^{18}$ Although ANN is a data-driven model, it nevertheless has been successful to learn from smaller data sets, achieving higher performance when predicting outputs, compared to probabilistic models such as Bayesian networks or decision trees. ${ }^{14,19}$ ANN is capable of finding complex multidimensional relationships between inputs and outputs of the system and has been a proven particular suite to the general field of bioinformatics. Evidently, it has great potential in bone tissue engineering decision support that can monitor and predict the outcome of clinical problems and provide valuable information to both clinicians and tissue engineers, thus reducing failures and improving therapeutic effects. The latter is often engaged in the challenging task of modelling and designing hierarchical synthetic scaffolds for skeletal regeneration. ${ }^{20-23}$ For trabecular hard tissue engineering, in particular, the application of neural networks is novel and largely an unexplored area. There are some reports of trabecular bone modelling, but only few studies adopted ANN-based approach ${ }^{24,25}$ in combination with more established computational techniques of FEA of the trabecular bone as a cellular structure. For example, ANN has been applied successfully to predict the mechanical properties of trabecular bone, ${ }^{24}$ and estimated the apparent fatigue damage accumulation due to cyclic loading in a trabecular bone. The promise of ANN for modelling the mechanical behaviour of trabecular bone was demonstrated ${ }^{25}$ by estimating load from the spatial distribution of density in the femoral bone. The key limitations of both studies were the dependence of the ANN performance on the validity of the assumptions in the corresponding FEA model. It was evident ${ }^{24,25}$ therefore that further work in this area using ANN is vital. A strategic approach must consider clinically important parameters such as age, gender, anatomical bone location, biological factors, pore morphology of the trabecular network structure and the effect of drugs prescribed to patients. Literature in this field is scarce and supports the view that there is an urgent need for an effective stand-alone ANN-based datadriven algorithms that integrate complex structural and mechanobiological parameters in a single model of human trabecular tissue. In parallel, understanding how various structural (3D constructs) and mechanical parameters correlate with the age and gender of the patients affected by bone loss can provide valuable clinical insights for diagnosis, prevention and potential treatment of bone disorders.

Age correlation with individual physical parameters of trabecular bone affected by osteoarthritis was investigated ${ }^{26}$ by applying bivariate linear regression model. The research has shown that no significant correlation exists between the age of the specimen and any of the specified mechanical property parameters for either gender. Also, by generalising for all osteoarthritis-affected cases, the structural parameters and mechanical strength of the cancellous bone did not depend on age for patients suffering from this disorder. The authors hypothesized that the correlation of the age with the individual mechano-biological parameters, when studied in isolation, could potentially result in the loss of valuable inter-dependence of all the variables. The use of a simple bivariate regression for data with multi-variate dependency ${ }^{27}$ can lead to false-negative results whereas multi-variable regression analysis is more appropriate when such dependency may exist.

Multi-variable regression analysis implies investigation of how several parameters simultaneously influence the desired output. It also allows the prediction of an outcome (output) of a system based on values of a set of predictor variables (inputs), which can be measured in the experiments or represent clinical data. Several computational modelling studies of mechanical properties of the trabecular bone ${ }^{28,29}$ have shown that a theoretical mathematical approach can be applied to determine the effects of scaffold micro-architecture on new bone formation. This comprehensive model allowed successful theoretical simulation of bone regeneration and the scaffold mass loss over time. However, the work highlighted the difficulty in obtaining the initial input parameters and thus the model required further extensive experimental validation for its use in practical scaffold design applications. Furthermore, experimentally derived specific mechanical properties, such as compressive strength, hardness and Young's modulus of trabecular bone, were used. ${ }^{28,29}$ These studies provided valuable and experimentally validated conclusions regarding compressive properties of the trabecular bone; however, the complexity of the experiments failed to consider the mechanical properties in conjunction with the essential structural and mechanobiological parameters of the trabecular bone behaviour for its use in hard tissue engineering. Therefore, the current state of research on trabecular bone modelling demands a more comprehensive model that could integrate in a single system with various mechanical, structural and biological aspects of the trabecular bone and yet be based on the actual experimental/clinical data.

The aim of this work is to apply ANN approach to perform multidimensional data analysis of physical and mechanical properties of trabecular bone and investigate correlation of the properties with age of male and female specimens. The research targets a particular type of cancellous bone, which has been affected by osteoarthritis. Such computational approach is largely unexplored and original in the area of tissue engineering and could provide a sound platform that integrates machine learning and tissue engineering.

\section{Materials and methods}

\subsection{Data}

This work focuses on a particular data set of 35 samples of four mechanical parameters for male and female specimens of various ages, adapted from a study on cancellous bones affected by osteoarthritis. ${ }^{26}$ Trabecular tissue samples from the femoral head of 37 patients suffering from severe osteoarthritis and undergoing total hip arthroplasty were considered. For each trabecular bone sample, the series of experiments involving micro-computed tomography (micro-CT) scanning at the isotropic pixel resolution of $19.5 \mu \mathrm{m}$ with a complete rotation over $185^{\circ}$, deformation testing with extensometer code to the sample and ashing at $650^{\circ} \mathrm{C}$ for $24 \mathrm{~h}$ with 
Bioinspired, Biomimetic and Nanobiomaterials Volume 4 Issue BBN1
Neural networks for analysis of trabecular

bone in osteoarthritis

Khovanova, Shaikhina and Mallick subsequent mass measurements were conducted. During selection of this primary data, care was taken to ensure that samples from the patients affected by secondary osteoarthritis and other bone and joint diseases were excluded from the experiments. The tissue samples were extracted from each femur to ensure consistency of shape, location and alignment. The cylindrically shaped fragment ( $20 \mathrm{~mm}$ in free height and $10 \mathrm{~mm}$ in diameter) of cancellous bone was chosen from the principal compressive region of the femoral head and positioned for extraction so that the cylinder axis was aligned with the fixed main trabecular direction for each specimen.

These data were extracted from original measurement ${ }^{26}$ comprising a total of 35 bone samples: 17 male and 18 female with age ranging between 42 and 87 . The following four mechanical and structural parameters were extrapolated from the graphs: compressive strength (in MPa) measured by extensometer in deformation testing, porosity $(\%)$ characterised by the bone volume fraction $\mathrm{BV} / \mathrm{TV}$ (derived from the cross-sectional micro-CT images by dividing the sum of voxels identified as trabecular bone by the sum of voxels composing the total volume of interest), level of inter-connectivity indicated by the trabecular thickness factor Tb.Th (dimensionless: measured in micro-CT using spherical estimation), morphology characterised by the Structure Model Index, SMI (dimensionless: determined from micro-CT images by approximating the bone topology in terms of rods and plates). The precision error of the data extraction is less than $0.7 \%$ for any given sample.

The final data set (Table 1) comprised five-dimensional matrix of one gender indicator coupled with the four mechanical parameters listed above as the input and the vector of specimens age as the corresponding output.

\subsection{Modelling}

\subsubsection{ANN configuration and data input}

Figure 1 illustrates the architecture of a multi-layer perceptron feedforward network adapted from Haykin. ${ }^{30}$ Five input neurons process the input vector of compressive strength, porosity, gender, morphology and inter-connectivity level in the trabecular bone samples: one hidden layer containing seven neurons maps the inputs to the corresponding output, and one output neuron produces the final calculation of the age of the specimen. The neurons are arranged in layers, with outputs from neurons in one layer feeding into the inputs of all the neurons in the next layer. The ANN was developed in MATLAB R2012b using Neural Network Toolbox 8.0.

The process of modelling was divided in three stages: training of ANN, validation and testing of the results. For this purpose, three data sets were required, and therefore, available 35 samples were randomly divided into three sets of training, validating and testing data.

\subsubsection{Training and validation of ANN}

Levenberg-Marquardt backpropagation algorithm was used ${ }^{31}$ to train data set available for this study, as the learning configuration of this algorithm is most commonly used with multi-layer perceptrons..$^{32}$ The algorithm is based on gradient descent method to calculate and update the values of weights and biases in the network, by using a function optimisation technique to reduce the error in the training data set. Notably, Levenberg-Marquardt algorithm is particularly efficient in minimising sum of square errors for non-linear functions, and therefore its performance has been measured by root mean squared error (rmse). ${ }^{33}$

1. $\quad$ rmse $=\sqrt{\frac{\sum_{i=1}^{n}\left[y_{i}-\hat{y}_{i}\right]^{2}}{n}}$

where $y_{i}$ is the actual output, $\hat{y}_{i}$ is the output predicted by the ANN and $n$ is the total number of samples. Five input variables, forming the ANN training set, were linearly independent as this condition maximises the learning efficiency of the backpropagation algorithm. ${ }^{18}$

To avoid overfitting, ${ }^{34}$ the model used a training routine incorporating principles from cross-validation, allowing monitoring the change in error for a separate set of validation data, ${ }^{35}$ and early-stopping technique. The latter significantly reduces the validation error while allowing training error to reach a minimum without overfitting ${ }^{36}$ (Figure 2(a)).

\subsubsection{Performance of the model}

The predictive power of the ANN model has been quantified by the linear regression factor $R$ between the predicted and actual output values of age and was chosen as the primary statistical indicator of the model quality. ${ }^{37}$

2.

$$
\text { rmse }=\sqrt{1-\frac{\sum_{i=1}^{n}\left[y_{i}-\hat{y}_{i}\right]^{2}}{\sum_{i=1}^{n}\left[y_{i}-\bar{y}_{i}\right]^{2}}}
$$

where $\bar{y}_{i}$ is the mean of $y_{i}$, and $0<R<1 . R=1$ corresponds to a perfect fit signifying that $100 \%$ of the variance in the dependent output variable can be explained by the regression equation. ${ }^{37} \mathrm{In}$ addition, linear regression factor shows whether the values of rmse (Equation 1) obtained as the result of ANN training can be used for characterising the quality of training. A satisfactory qualitative value of $R$ varies substantially depending on the specific application and purpose of the predictive model. Based on indications reported in the literature, ${ }^{37,38}$ the ANN approach for correlation analysis of the trabecular hard tissue, developed here for the first time, assumed the following benchmark interpretations of the regression factor: $R>0.96$ for very high predictive performance, $0.86<R<0.96$ for 
Bioinspired, Biomimetic and Nanobiomaterials Volume 4 Issue BBN1
Neural networks for analysis of trabecular bone in osteoarthritis

Khovanova, Shaikhina and Mallick

\begin{tabular}{|c|c|c|c|c|c|c|}
\hline Sample & $\begin{array}{l}\text { Age: } \\
\text { years }\end{array}$ & Porosity: \% & $\begin{array}{c}\text { Gender } \\
\text { (1 - female, } \\
0 \text { - male) }\end{array}$ & $\begin{array}{c}\text { Morphology } \\
\text { (SMI) }\end{array}$ & $\begin{array}{l}\text { Level of inter- } \\
\text { connectivity } \\
\text { (Tb.Th) }\end{array}$ & $\begin{array}{l}\text { Compressive } \\
\text { strength: } \mathrm{MPa}\end{array}$ \\
\hline 1 & $41 \cdot 8$ & $32 \cdot 48$ & 1 & 0.06 & 243 & $20 \cdot 9$ \\
\hline 2 & 49 & $9 \cdot 68$ & 1 & 1.93 & 154 & $8 \cdot 22$ \\
\hline 3 & 52 & $21 \cdot 55$ & 1 & 1.42 & 224 & 6.91 \\
\hline 4 & 57 & $26 \cdot 56$ & 1 & 0.48 & 239 & $18 \cdot 2$ \\
\hline 5 & $60 \cdot 9$ & 33.84 & 1 & 0.31 & 252 & $20 \cdot 5$ \\
\hline 6 & $62 \cdot 8$ & $22 \cdot 48$ & 1 & 0.7 & 283 & $12 \cdot 2$ \\
\hline 7 & $63 \cdot 9$ & $43 \cdot 54$ & 1 & -0.82 & 212 & $9 \cdot 46$ \\
\hline 8 & 64 & $17 \cdot 89$ & 1 & $1 \cdot 22$ & 419 & $23 \cdot 1$ \\
\hline 9 & 66 & $25 \cdot 34$ & 1 & 0.92 & 263 & $15 \cdot 4$ \\
\hline 10 & $67 \cdot 1$ & $27 \cdot 59$ & 1 & 0.64 & 223 & $19 \cdot 4$ \\
\hline 11 & $68 \cdot 1$ & $9 \cdot 82$ & 1 & $2 \cdot 1$ & 197 & $2 \cdot 76$ \\
\hline 12 & $69 \cdot 9$ & $39 \cdot 72$ & 1 & -0.43 & 299 & $23 \cdot 2$ \\
\hline 13 & $71 \cdot 5$ & $26 \cdot 89$ & 1 & $0 \cdot 38$ & 367 & $18 \cdot 9$ \\
\hline 14 & $72 \cdot 6$ & $13 \cdot 72$ & 1 & 1.59 & 247 & 1.93 \\
\hline 15 & $73 \cdot 9$ & $20 \cdot 99$ & 1 & $1 \cdot 04$ & 239 & $8 \cdot 15$ \\
\hline 16 & 74.9 & $15 \cdot 36$ & 1 & $0 \cdot 8$ & 218 & $6 \cdot 49$ \\
\hline 17 & 76 & 24.98 & 1 & 0.54 & 314 & $17 \cdot 8$ \\
\hline 18 & 87 & $32 \cdot 41$ & 1 & 0.3 & 326 & $24 \cdot 2$ \\
\hline 19 & $41 \cdot 7$ & $30 \cdot 40$ & 0 & -0.17 & 287 & $21 \cdot 5$ \\
\hline 20 & $45 \cdot 7$ & $27 \cdot 45$ & 0 & $0 \cdot 45$ & 257 & $19 \cdot 6$ \\
\hline 21 & $46 \cdot 8$ & $35 \cdot 59$ & 0 & -0.05 & 288 & $24 \cdot 3$ \\
\hline 22 & $47 \cdot 9$ & $36 \cdot 98$ & 0 & $-0 \cdot 31$ & 284 & $16 \cdot 4$ \\
\hline 23 & $49 \cdot 8$ & 38.71 & 0 & 0.04 & 265 & $11 \cdot 1$ \\
\hline 24 & $49 \cdot 7$ & $22 \cdot 68$ & 0 & 0.82 & 244 & $26 \cdot 5$ \\
\hline 25 & $62 \cdot 9$ & $27 \cdot 51$ & 0 & $0 \cdot 44$ & 266 & $18 \cdot 5$ \\
\hline 26 & $64 \cdot 9$ & $26 \cdot 64$ & 0 & $0 \cdot 39$ & 246 & $19 \cdot 3$ \\
\hline 27 & $65 \cdot 8$ & $37 \cdot 56$ & 0 & -0.23 & 303 & $28 \cdot 8$ \\
\hline 28 & 68 & $12 \cdot 16$ & 0 & $0 \cdot 71$ & 178 & 14 \\
\hline 29 & 68 & $25 \cdot 34$ & 0 & $1 \cdot 77$ & 219 & 4.91 \\
\hline 30 & $72 \cdot 9$ & $17 \cdot 44$ & 0 & $1 \cdot 33$ & 261 & $9 \cdot 81$ \\
\hline 31 & $73 \cdot 9$ & $29 \cdot 75$ & 0 & 0.04 & 307 & $23 \cdot 7$ \\
\hline 32 & $77 \cdot 8$ & 32.08 & 0 & $0 \cdot 15$ & 270 & $22 \cdot 2$ \\
\hline 33 & $81 \cdot 8$ & $31 \cdot 64$ & 0 & $0 \cdot 36$ & 271 & $24 \cdot 4$ \\
\hline 34 & 84.9 & $21 \cdot 76$ & 0 & $0 \cdot 7$ & 234 & $13 \cdot 3$ \\
\hline 35 & 87 & $19 \cdot 42$ & 0 & 1.08 & 193 & $9 \cdot 12$ \\
\hline
\end{tabular}

Table 1. Secondary data on 35 bone samples 
Bioinspired, Biomimetic and Nanobiomaterials Volume 4 Issue BBN1
Neural networks for analysis of trabecular

bone in osteoarthritis

Khovanova, Shaikhina and Mallick strong correlation with good predictive ability and $0.6<R<0.86$ for statistically significant correlation with weak predictive value.

\section{Results}

\subsection{Training and validation}

Twenty samples were randomly chosen for ANN training, five further samples for validation and last ten were used as testing data set. Applying Levenberg-Marquardt algorithm, ${ }^{32}$ the ANN was trained by altering the weights of neurons and biases

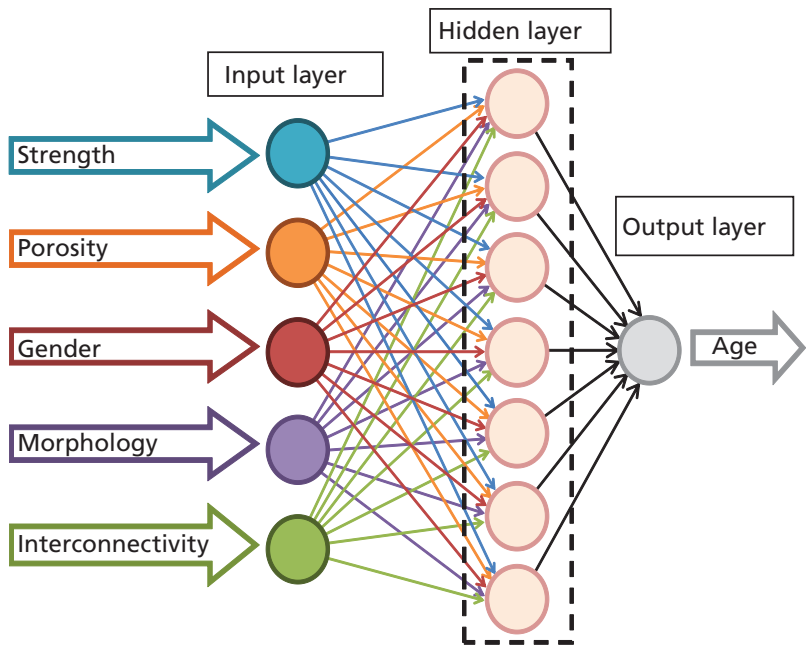

Figure 1. Neural network architecture defining the input, hidden and output layers, where each neuron is represented by a circle and the arrows denote the weight of the connection

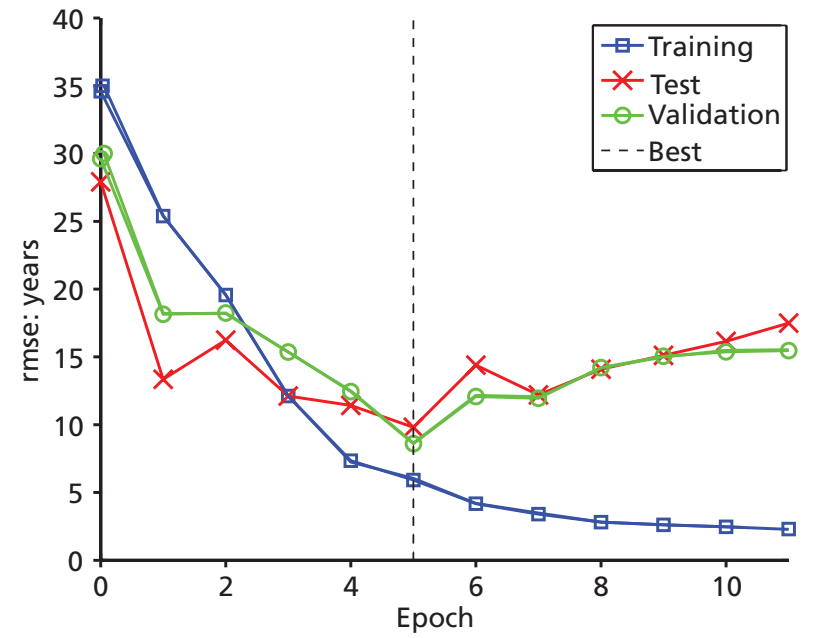

(a)

Figure 2. (a) ANN performance and (b) optimised ANN performance in training (blue line, marker ' $\square$ '), validation (green line, marker ' $o$ ') and testing (red line, marker ' $x$ ') as measured by rmse. Minimum validation error occurred at fifth epoch with $r_{\text {mse }}=9.81$ years for according to the data inputs. Each of the 20 samples was fed, its weights and biases were re-calculated and performance evaluated by validation. Following first iteration (epoch), samples were randomly shuffled and presented to the ANN once again with new values of parameters, obtained from the first epoch. This iterative procedure was repeated. It should be noted that in the process of a successful training, values of rmse tend to zero $\lim$ rmse $_{t r} \rightarrow 0$ , where index ' $t r$ ' indicates that rmse characterises training data set, while validation error had to be minimized not allowing over-fitting as verified by early-stopping technique. After five consecutive iterations (epochs) and validation checks, the least root mean squared error rmse $_{\text {val }}$, with which ANN could map the inputs to the target age over the validation samples, was found to be equal to 9.81 years (Figure 2(a)). On further iteration, the training error continued to decrease as expected as the system was progressively learning from the data (blue line with squares in Figure 2(a)). Validation error, however, showed its minimum value at the fifth epoch (green line with circles, Figure 2(a)), and continued training process past fifth epoch resulted in overfitting of the ANN.

Consequently, the ability of ANN to generalise data decreased causing an increase in the validation error. Thus, cross-validation was successfully utilized to monitor and establish the maximum performance of the ANN, that is, the optimal point with minimum validation error, and subsequently terminate training and revert the state of the ANN neurons to that optimum point. The accuracy of

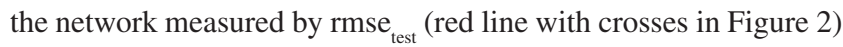
showed the value of 8.6 years at the optimal fifth epoch (red line with crosses, Figure 2(a)).

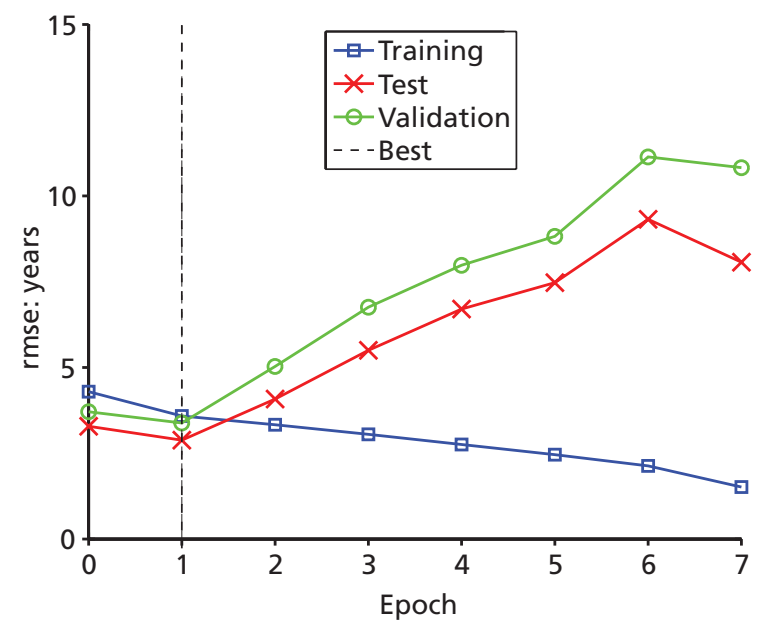

(b)

(a) and at first epoch with rmse $_{\text {val }}=2.87$ years for (b). Vertical dashed lines mark epoch numbers with minimal validation errors $\mathrm{rmse}_{\text {val }}$ ANN, artificial neural network; rmse, root mean squared error 
Bioinspired, Biomimetic and Nanobiomaterials Volume 4 Issue BBN1
Neural networks for analysis of trabecular

bone in osteoarthritis

Khovanova, Shaikhina and Mallick
The ANN performance (Figure 2(a)) measured by the regression factor $R$ was as follows: $R_{t r}=0.91$ for training data ( 20 samples) and $R_{\text {val }}=0.61$ for validation data (five samples). The ANN performed significantly better on the training data set, than on the validation samples, as the ANN was supplied with the target age values multiple times throughout the learning process. In addition, the validation set at only five input vectors comprised substantially less data points. This increased the effect of random distribution and outliers. The subsequent analysis of the ten samples reserved for testing arrived at regression factor $R_{\text {test }}=0 \cdot 87$. This indicates a strong positive correlation between predictive and target values of age and corresponds to a good predictive performance of the model (as specified by the benchmarks in Section 2.2.3). The regression factor calculated for the entire data set (35 samples) was $R_{\text {all }}=0 \cdot 86$. Overall, all four regression factors were within satisfactory boundaries indicating a strong positive correlation between the age and the mechanical parameters of the trabecular bone when multi-dimensional inter-dependencies between the parameters are considered.

\subsection{Optimisation of ANN}

ANN performance varied substantially with the random order with which the input data could be split into training, validation and test groups, as well as the sequence of the training. This is due to the limited size of the sample set but despite the potential drawback of increased ANN design complexity, this provided an opportunity for optimisation of the ANN.

In the context of data-driven systems, a statistical model can be optimised for the data it operates. Such optimisation offers vast potential to maximise the accuracy of the model and ability to fit the data. Contrarily, it can induce negative effects such as decreasing the ability of the system to generalise for the new data making the application of the model limited to the initial data set. This can however be resolved if the data set is fully and uniquely representative of all the data for which the model would be optimised. Hence, assuming that this pre-condition holds true for the entire range of trabecular bone samples, from the femoral head of osteoarthritis-affected patients, it was possible to optimise the ANN model and achieve enhanced predictive power, performance and generalisation ability.

Direct optimisation is generally achieved if the ANN is trained, validated and tested in a specific order and by the set number of the input data samples maximising its performance. ${ }^{14}$ However, determining this optimum order requires searching through all the possible combinations for 35 available data samples, randomly divided every time into three sets of groups: training data, validation samples and test vectors. For example, the number of possible combinations for 22 training data samples (comprising 63\% of all samples), seven validation (20\%) and six test (17\%) vectors is approximately $10^{33}$, which would require $10^{21}$ millennia to assess the performance of the ANN in MATLAB. Due to this constraint, the number of input combinations was reduced: a total of 2079 ANN were produced with random split and sequence in order to find the optimum. Each ANN was evaluated for the regression in an automated manner, and the best-performing ANN was determined.

The best validation results (Figure 2(b)) with optimised ANN occurs as early as during the first epoch, and the training was terminated at that instance. The regression factors between the target and output values of age, achieved by the optimised ANN (Figure 3) were improved in comparison with non-optimal case: $R_{t r}$ $=0.96$ for training data (22 samples, (Figure 3(a)), $R_{\text {val }}=0.99$ for validation data (seven samples, (Figure 3(b))) and $R_{\text {test }}=0.91$ for testing data (six samples, Figure 3(c)) with $R=0.96$ across the 35 samples (Figure 3(d)). Similarly, accuracy of the ANN measured by $\mathrm{rmse}_{\text {test }}$ (red line with crosses in Figure 2) has significantly increased: $\mathrm{rmse}_{\text {test }}=8.6$ years for initial ANN (at optimal fifth epoch, (Figure 2(a)), rmse $_{\text {test }}=3.37$ years for optimised ANN (at optimal first epoch, (Figure 2(b)).

These results re-iterate the existence of a strong positive multidimensional correlation between the age of the specimens of both genders and the mechano-biological parameters of their trabecular bones. The results also indicate high accuracy and predictive power of the optimised ANN.

Furthermore, a parameter sensitivity analysis was undertaken to assess whether or not it was possible to predict the age of a patient using less than five physical parameters of trabecular bone and yet retain the comparable level of modelling accuracy and predictive power of ANN. This was carried out by identifying which parameter out of the five inputs was least responsible for the correlation with age, as measured by the regression factor. If there was one such parameter, it could be potentially excluded from the input vector thus reducing the dimensionality of the multi-dimensional problem without losing any information on mechano-biological properties of trabecular bone.

Linear-independent component analysis is not directly applicable in multi-dimensional space, which necessitates alternative techniques to be used. ${ }^{27,39}$ It is suggested here that in the same ANN system, with the original input data set, the parameter in question is masked by substituting with its mean value across all the samples. By comparing the regression factors with the original factor $R_{\text {test }}=0.87$, it was determined whether or not the input parameter considered played a primary role in the correlation model.

The correlation was first tested with regard to the compressive strength masked by its mean value. It is shown (Figure 4) that the new linear regression trend (solid line, Figure 4(b)) has substantially deviated from the perfect fit (dashed line, Figure 4(b)) corresponding to the scenario where the ANN predictions (age 
Bioinspired, Biomimetic and Nanobiomaterials Volume 4 Issue BBN1
Neural networks for analysis of trabecular

bone in osteoarthritis

Khovanova, Shaikhina and Mallick

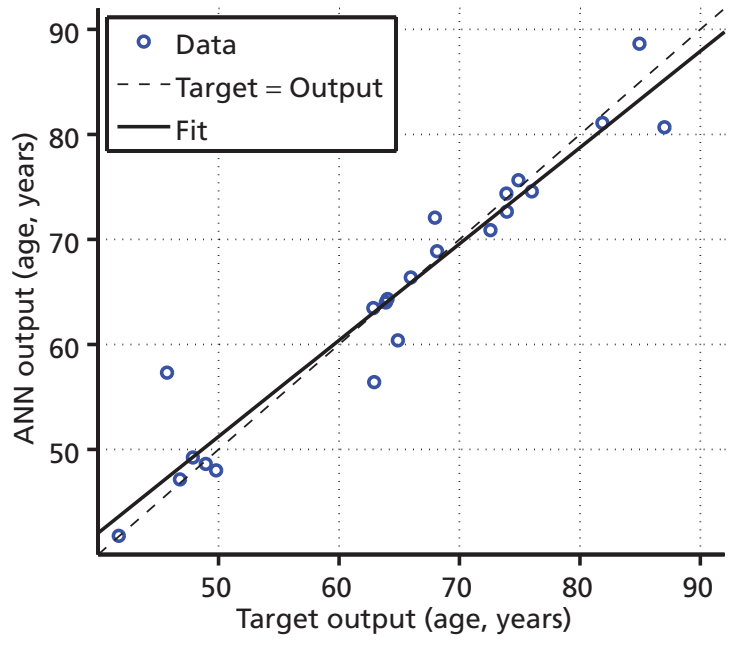

(a)

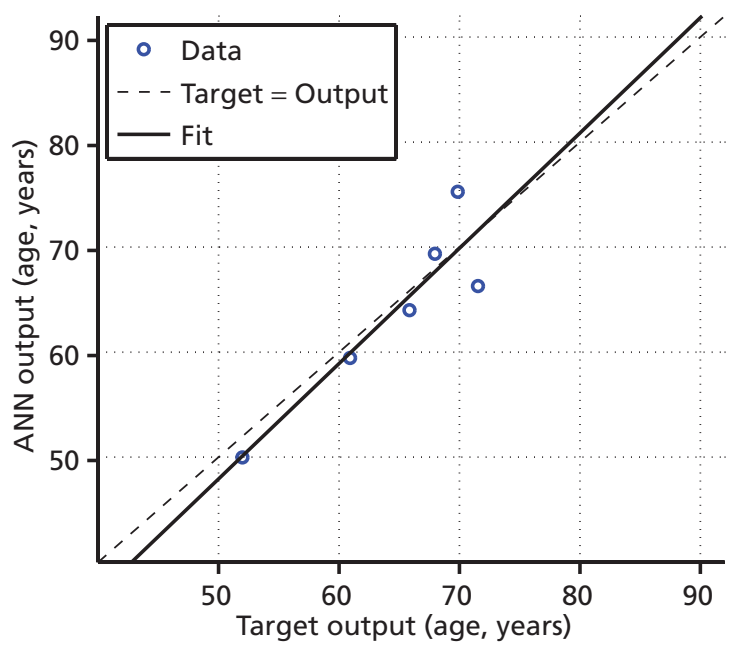

(c)

Figure 3. Optimised ANN: linear regression between the outputs and target value over (a) training, (b) validation, (c) test samples and (d) entire data set. Circles represent experimental data, solid

output) are equal to the actual age targets. The predictive power of the ANN reduced markedly as the regression factor between the target and the predicted age decreased from $R_{\text {test }}=0.86$ (Figure 4(a)) to $R_{\text {test }}=0.25$ (Figure 4(b)). Therefore, the impact of compressive strength on the overall correlation of the ANN model is statistically significant.

This significance was also quantified by the relative change of $71 \%$ in the regression factor (Table 2) demonstrating that compressive strength was responsible for $71 \%$ of the predictive power of the ANN model. Similarly, the above procedure was applied to the four remaining input parameters: the trabecular bone porosity, morphology, level of inter-connectivity and the gender

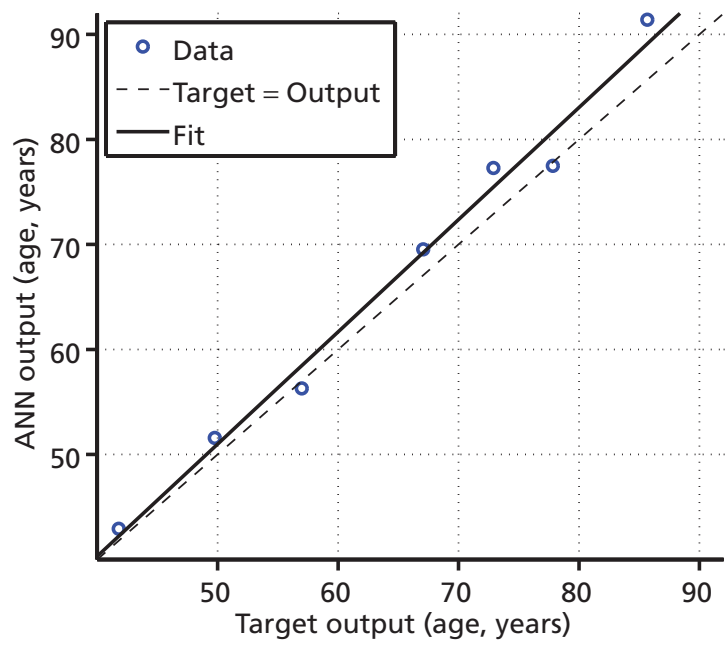

(b)

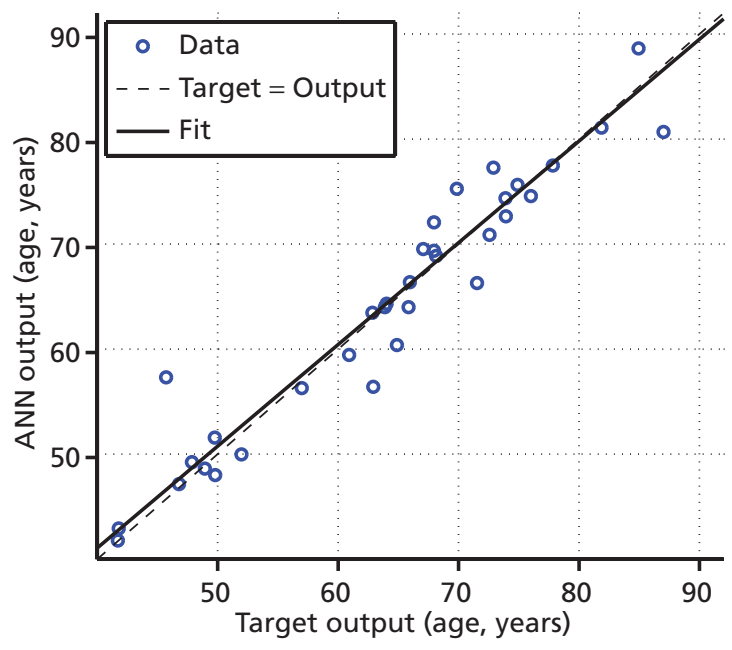

(d)

lines correspond to linear fitting and dashed lines correspond to the scenario when the ANN predictions (age output) are equal to the actual age targets. ANN, artificial neural network

of the specimens. It was found (Table 2) that morphology of the trabecular bone (SMI index) and the gender of the specimen are next most important components with 45 and $41 \%$ of the overall predictive power, respectively. The trabecular thickness (bone inter-connectivity), masked by its mean value, decreased the ANN predictive power by $35 \%$. Finally, porosity (bone volume fraction) was found to be the least important component with $27 \%$ of the predictive power.

As seen from Table 2, the regression factor does not exceed $0 \cdot 6$ benchmark, $R<0 \cdot 6$ (except for the porosity, discussed in Section 3), demonstrating weak predictive ability of the model and absence of statistically significant correlations between target and output 
Bioinspired, Biomimetic and Nanobiomaterials Volume 4 Issue BBN1
Neural networks for analysis of trabecular bone in osteoarthritis

Khovanova, Shaikhina and Mallick

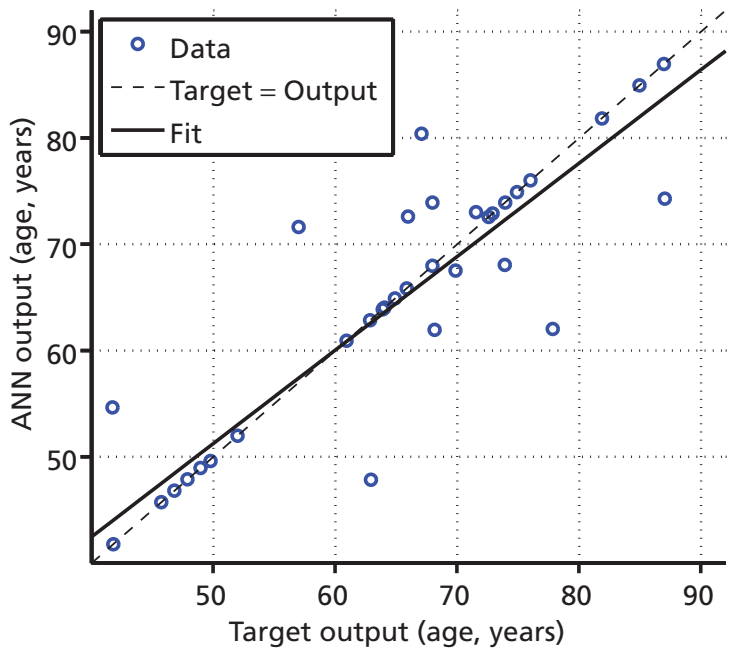

(a)

Figure 4. ANN output against target age with all five inputs ((a), $R_{\text {test }}$ $=0.86)$ and with the compressive strength input masked $\left((b), R_{\text {test }}=\right.$ $0 \cdot 25)$. Circles represent experimental data, solid lines correspond to

\begin{tabular}{lccccc} 
& \multicolumn{2}{c}{$\boldsymbol{R}_{\text {test }}$} & & \multicolumn{2}{c}{ Change in $\boldsymbol{R}_{\text {test }}$} \\
\cline { 2 - 3 } \cline { 5 - 6 } Input parameter & Initial & New & & Absolute & $\begin{array}{c}\text { Relative: } \\
\%\end{array}$ \\
\hline $\begin{array}{l}\text { Compressive } \\
\text { strength: MPa }\end{array}$ & 0.86 & 0.25 & & 0.61 & 71 \\
$\begin{array}{l}\text { Porosity: \% } \\
\text { Morphology (SMI) }\end{array}$ & 0.86 & 0.63 & & 0.23 & 27 \\
$\begin{array}{l}\text { Level of inter- } \\
\text { connectivity (Tb.Th) }\end{array}$ & 0.86 & 0.56 & & 0.30 & 45 \\
Gender & 0.86 & 0.51 & & 0.35 & 41 \\
\hline
\end{tabular}

Table 2. Sensitivity analysis results for each input parameter

values of age when one of the five parameters is excluded from the consideration. Thus, none of the five input parameters can be compromised for dimensionality reduction of the model, and age of the specimen depends on all five mechano-biological parameters of the trabecular bone: compressive strength, porosity, trabecular thickness and morphology for specimens of both genders.

\subsection{Compressive strength model}

A single input-output inversion enables the ANN model to be used for determining compressive strength of a trabecular tissue from the bone volume fraction, its SMI and trabecular thickness parameters in combination with the age and the gender of the specimen. To validate the effect of such design alteration, the ANN was fed with the age values as one of the input parameters, and assigning compressive strength to the output neuron.

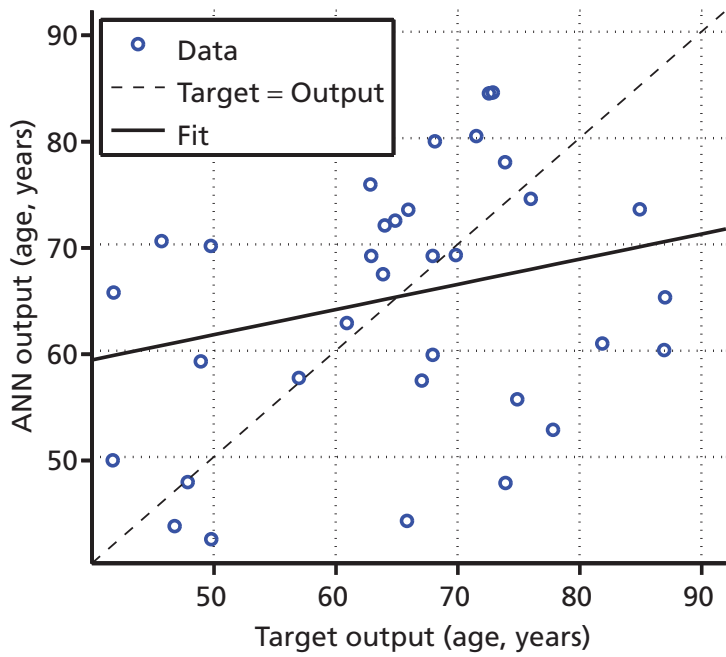

(b)

linear fitting and dashed lines correspond to the scenario when the ANN predictions (age output) are equal to the actual age targets. ANN, artificial neural network

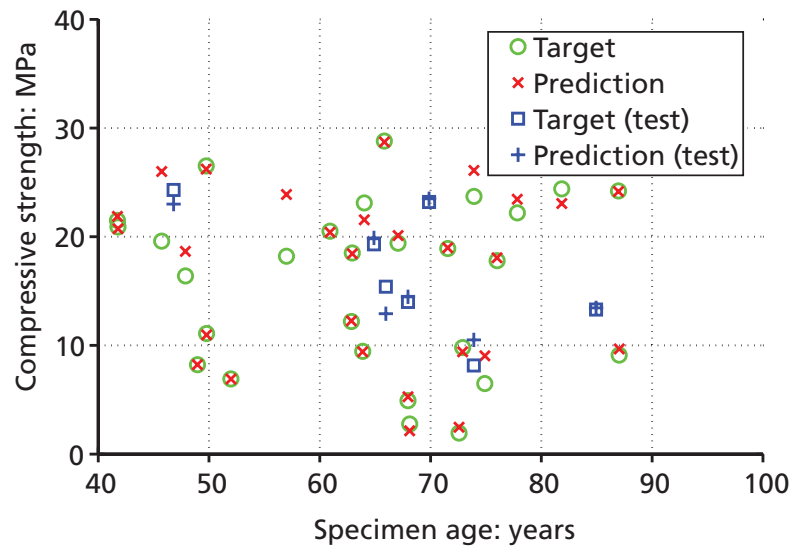

Figure 5. Scatter plot of target (circles, squares) and predicted (crosses, pluses) compressive strength values for all samples (distributed by age of the specimen). Results corresponding to test samples are shown by blue squares and pluses

Subsequently, the modified ANN was trained, validated, optimised and tested using the methodology presented in the previous sections. It was found that the optimal ANN is able to predict the compressive strength of a bone sample with a standard error rmse $=1.90 \mathrm{MPa}$ (Figure 5). The regression factors between the target and output strength values are $R_{t r}=0.998$ for training, $R_{\text {val }}$ $=0.90$ for validation and $R_{\text {test }}=0.97$ for testing data with $R=0.97$ across the entire data set. The high accuracy of the compressive strength model enables estimation of bone fracture risk from the biological and structural parameters that are measured without invasive tests on the specimen. 
Bioinspired, Biomimetic and Nanobiomaterials Volume 4 Issue BBN1
Neural networks for analysis of trabecular

bone in osteoarthritis

Khovanova, Shaikhina and Mallick

\section{Discussion}

The authors applied ANN in order to account for possible complex inter-dependency of the mechano-biological parameters of trabecular bone affected by osteoarthritis. A generalized ANN model has been presented to be capable of predicting the output age from the physical parameters of the bone. It is important to note that ANN generally do not require pre-defined causal relations between the model elements and the initial design assumptions do not depend on the exact problem formulations. ${ }^{19}$ This makes ANN the most applicable tool for the presented correlation analysis of the mechano-biological parameters of the trabecular bone based on a relatively small experimental data set.

When analysed independently in a multi-dimensional space, the porosity of the trabecular bone was found to be the least important factor influencing the age as an output of the model (Table 2), whereas the compressive strength was the most important of the ANN inputs. At first observation, it may seem surprising that in the absence of the porosity the correlation of age with the remaining parameters decreases only by $27 \%$. This is attributed to porosity being commonly regarded as one of the key factors associated with age of patients suffering from bone disorders. ${ }^{26,40}$ However, this finding can be explained by re-calling the fact that for this particular data set, the bone volume fraction was used as a measure of both porosity and indirectly the apparent density. Apparent density, in turn, correlates with the compressive strength: Gibson ${ }^{41}$ quantified this relationship using a mechanical model of cellular solids and stated that compressive strength of the trabecular bone depends on apparent density raised to the $3 / 2$ power. Such mathematical formulation extends to the trabecular bone samples used in this study since the experimental results ${ }^{26}$ demonstrated that compressive strength correlates with the porosity of diseased trabecular bone in polynomial manner with regression factor $R=$ 0.97 . Therefore, the compressive strength input in the proposed ANN supersedes the porosity input in terms of its impact on predicting the age.

The computational model of trabecular hard tissue developed here successfully integrates the primary structural, mechanical and biological properties and has potential to be of particular value to hard tissue engineers when designing bioscaffolds that imitate the natural trabecular bone. It is thus possible to predict how compressive strength, bone volume fraction, trabecular thickness and SMI are inter-dependent of age and gender, and tissue engineers could then tailor the scaffold designs for an individual patient to match the mechano-biological properties of the trabecular tissue at the site of implantation.

Assuming the data set is considered representative of the entire population of trabecular hard tissue affected by bone loss, this model provides a powerful predictive tool for a general set of clinical applications, including cases of osteoarthritis, osteopenia or osteoporosis for patients of a wide age spectrum, gender and varying severity of the disease. Although the study is somewhat limited by the relatively small size of tissue samples available, it has nonetheless confirmed the ability of such data to support the ANN approach in a clinical setting. Since the system is data driven, the model can be considered as a continuum since it processes the new data once they become available. This model, with its underlying machine-learning algorithms, is designed to improve its generalisation with every new data sample..$^{42,43}$ The ability of this model to evolve with additional training and to simulate any given data makes this ANN model a promising versatile tool for a range of hard tissue engineering and clinical applications such as hard issue augmentation.

The trabecular bone model developed in this study serves as a foundation for further research on applications of ANN and other machine-learning algorithms for data-driven modelling in hard tissue engineering. The next step will naturally involve simulation of the model on the extended set of input data as could become available through the on-going experimental research on synthetic scaffolds ${ }^{20-22}$ or primary and secondary clinical data. As a datadriven model, the ANN was so designed as to learn from the input data and can be trained to yield predictive results for not only osteoarthritis-affected samples but a variety of other clinical cases including osteoporosis and metastatic cancer for early diagnosis of the patients of both genders, varying age and the disease severity. This strategy can be further extended to scope problems by investigating other mechano-biological inter-dependencies on the live tissue-implant interface in the trabecular bone from the human femoral head or, alternatively, model the hard tissue from other anatomical locations. Such research would substantially increase range of potential clinical applications of the computational models in biomedicine and orthopaedics.

For hard tissue engineers, it would be of a particular value to model the inverse problem: for instance, how compressive strength of the trabecular tissue (ANN output) varies with various mechanobiological (input) parameters. The current model already laid a solid foundation for such study and a new model can be produced by reverting the current outputs and inputs (as above), once the appropriate experimental data become available. The potential benefit of such model is a tool that could aid tissue engineers to optimise the hard tissue scaffolds during initial stages of the design process.

\section{Conclusions}

The ANN model proposed in this study demonstrated that mechanical and structural properties of the trabecular bone depend on age for the osteoarthritis-affected patients. The model provided a tool for a multi-variate non-linear regression analysis and indicated that combined changes in the individual bone properties such as porosity, compressive strength, level of interconnectivity and morphology cause the bone aging. The resultant 
Bioinspired, Biomimetic and Nanobiomaterials Volume 4 Issue BBN1
Neural networks for analysis of trabecular

bone in osteoarthritis

Khovanova, Shaikhina and Mallick
ANN model is capable of determining the age of a specimen from the parameters with standard error of 8.6 years. Such accuracy may not be sufficient for safely critical or high-precision applications; the proposed ANN can, however, be relied on as a basic decision support model for clinicians and tissue engineering practitioners even before optimisation of specific data set.

When optimised for the particular set of 35 samples by determining the best training split across a few thousands of possible combinations using iterative approach, the ANN achieved the regression factor of 0.96 across the entire data set and 0.91 for test samples. Its predictive power improved markedly compared to initial results, and the optimised ANN model was able to determine the age of the specimen from the input properties with rmse error of 3.37 years. The optimisation assumed that the original data set is fully representative of the all trabecular bone samples for which the model would be utilised.

Analysis of input components sensitivity implemented using ANN revealed that none of the five input parameters can be excluded from the model and therefore aging of the bone, affected by osteoarthritis, depends on all five mechano-biological properties of the trabecular bone: compressive strength, porosity, trabecular thickness and morphology for specimens of both genders. The compressive strength was found to be the most important factor in the correlation with age.

\section{Acknowledgements}

This study was supported by EPSRC UK (Grant EP/K02504X/1).

\section{REFERENCES}

1. Gibson, L. J.; Ashby, M. F.; Harley, B. A. Cellular Materials in Nature and Medicine. Cambridge: Cambridge University Press, 2010.

2. Galante, J.; Rostoker, W.; Ray, R. D. Physical properties of trabecular bone. Calcified Tissue Research 1970, 5, 236-246.

3. Hildebrand, T.; Laib, A.; Muller, R.; Dequeker, J.; Ruegsegger, P. Direct three-dimensional morphometric analysis of human cancellous bone: microstructural data from spine, femur, iliac crest, and calcaneus. Journal of Bone and Mineral Research: The Official Journal of the American Society for Bone and Mineral Research 1999, 14, 1167-1174.

4. Carter, D. R.; Hayes, W. C. Bone compressive strength: the influence of density and strain rate. Science 1976, 194, 1174-1176.

5. Hoffler, C. E.; Moore, K. E.; Kozloff, K.; Zysset, P. K.; Brown, M. B.; Goldstein, S. A. Heterogeneity of bone lamellar-level elastic moduli. Bone 2000, 26, 603-609.

6. Kopperdahl, D. L.; Keaveny, T. M. Yield strain behavior of trabecular bone. Journal of Biomechanics 1998, 31, 601-608.
7. Morgan, E. F.; Bayraktar, H. H.; Keaveny, T. M. Trabecular bone modulus-density relationships depend on anatomic site. Journal of Biomechanics 2003, 36, 897-904.

8. Crane, G. J.; Fazzalari, N. L.; Parkinson, I. H.; VernonRoberts, B. Age-related changes in femoral trabecular bone in arthrosis. Acta Orthopaedica 1990, 61, 421-426.

9. Ding, M.; Odgaard, A.; Linde, F.; Hvid, I. Age-related variations in the microstructure of human tibial cancellous bone. Journal of Orthopaedic Research 2002, 20, 615-621.

10. Kabel, J.; Van Rietbergen, B.; Odgaard, A.; Huiskes, R. Constitutive relationships of fabric, density, and elastic properties in cancellous bone architecture. Bone 1999, 25, 481-486.

11. Keyak, J. H.; Rossi, S. A. Prediction of femoral fracture load using finite element models: an examination of stress-and strain-based failure theories. Journal of Biomechanics $\mathbf{2 0 0 0 ,}$ 33, 209-214.

12. Silva, M. J.; Keaveny, T. M.; Hayes, W. C. Computed tomography-based finite element analysis predicts failure loads and fracture patterns for vertebral sections. Journal of Orthopaedic Research 1998, 16, 300-308.

13. Geris, L. Computational Modeling in Tissue Engineering. Berlin: Springer-Verlag, 2013.

14. Hudson, D. L.; Cohen, M. E. Neural Networks and Artificial Intelligence for Biomedical Engineering. New York: Institute of Electrical and Electronics Engineers, 2000.

15. Trmal, J.; Zelinka, J.; Müller, L. Adaptation of a feedforward artificial neural network using a linear transform. In Text, Speech and Dialogue (Sojka, P.; Horák, A.; Kopeček, I.; Pala, K. (eds.)). Berlin: Springer Berlin Heidelberg, 2010, 423-430.

16. Kursin, A. Neural network: input anticipation may lead to advanced adaptation properties. In Artificial Neural Networks and Neural Information Processing - ICANN/ICONIP 2003 (Kaynak, O.; Alpaydin, E.; Oja, E.; Xu, L. (eds.)). Berlin: Springer Berlin Heidelberg, 2003, 779-785.

17. Someren, M.; Hagen, S. Machine learning and reinforcement learning. In Do Smart Adaptive Systems Exist? (Gabrys, B.; Leiviskä, K.; Strackeljan, J. (eds.)). Berlin: Springer Berlin Heidelberg, 2005, 81-104.

18. Smith, M. Neural Networks for Statistical Modeling. New York: Van Nostrand Reinhold, 1993.

19. Hall, L. O.; Liu, X.; Bowyer, K. W.; Banfield, R. Why are neural networks sometimes much more accurate than decision trees: an analysis on a bio-informatics problem. IEEE International Conference 2003, 3, 2851-2856.

20. Mallick, K. K. Freeze casting of porous bioactive glass and bioceramics. Journal of the American Ceramic Society 2009 , 92, 85-94.

21. Mallick, K. K.; Winnett, J. Preparation and characterization of porous Bioglass ${ }^{\circledR}$ and PLLA scaffolds for tissue engineering applications. Journal of the American Ceramic Society 2012, 95, 2680-2686. 
Bioinspired, Biomimetic and Nanobiomaterials Volume 4 Issue BBN1
Neural networks for analysis of trabecular

bone in osteoarthritis

Khovanova, Shaikhina and Mallick
22. Mallick, K. K.; Winnett, J.; van Grunsven, W.; Lapworth, J.; Reilly, G. C. Three-dimensional porous bioscaffolds for bone tissue regeneration: fabrication via adaptive foam reticulation and freeze casting techniques, characterization, and cell study. Journal of Biomedical Materials Research Part A 2012, 100, 2948-2959.

23. Lee, S. J.; Atala, A. Scaffold technologies for controlling cell behavior in tissue engineering. Biomedical Materials 2013, 8, $1-2$.

24. Hambli, R. Apparent damage accumulation in cancellous bone using neural networks. Journal of the mechanical Behavior of Biomedical Materials 2011, 4, 868-878.

25. Zadpoor, A. A.; Campoli, G.; Weinans, H. Neural network prediction of load from the morphology of trabecular bone. Applied Mathematical Modelling 2013, 37, 5260-5276.

26. Perilli, E.; Baleani, M.; Öhman, C.; Baruffaldi, F.; Viceconti, M. Structural parameters and mechanical strength of cancellous bone in the femoral head in osteoarthritis do not depend on age. Bone 2007, 41, 760-768.

27. Hyvärinen, A.; Karhunen, J.; Oja, E. Independent Component Analysis. New York: Wiley, 2004.

28. Goulet, R. W.; Goldstein, S. A.; Ciarelli, M. J.; Kuhn, J. L.; Brown, M.; Feldkamp, L. The relationship between the structural and orthogonal compressive properties of trabecular bone. Journal of Biomechanics 1994, 27, 375-389.

29. Öhman, C.; Baleani, M.; Perilli, E.; Dall'Ara, E.; Tassani, S.; Baruffaldi, F.; Viceconti, M. Mechanical testing of cancellous bone from the femoral head: experimental errors due to off-axis measurements. Journal of Biomechanics 2007, 40, 2426-2433.

30. Haykin, S. Neural Networks: A Comprehensive Foundation. Upper Saddle River: Prentice Hall, 1999.

31. Wilamowski, B. M. Neural network architectures and learning algorithms. Industrial Electronics Magazine, IEEE 2009, 3, 56-63.

32. Hagan, M. T.; Demuth, H. B.; Beale, M. H. Neural Network Design. Pacific Grove: Brooks/Cole, 1996.

33. Wackerly, D. D.; Mendenhall, W.; Scheaffer, R. L. Mathematical Statistics With Applications. Belmont: Thomson Brooks/Cole, 2008.

34. Taylor, J. G. Neural Networks and Their Applications. UNICOM, 1996.

35. Stone, M. Cross-validatory choice and assessment of statistical predictions. Journal of the Royal Statistical Society Series B (Methodological) 1974, 111-147.

36. White, H. Artificial Neural Networks: Approximation and Learning Theory. Oxford: Blackwell, 1992.

37. Cohen, J.; Cohen, S. G.; West, W. C.; Aiken, L. S. Applied Multiple Regression: Correlation Analysis for the Behavioral Sciences, 3rd edn. Mahwah: Erlbaum, 2003.

38. Brause, R. W. Medical analysis and diagnosis by neural networks. In Medical Data Analysis. Heidelberg: Springer, 2001, 1-13.
39. Dong, D.; McAvoy, T. J. Nonlinear principal component analysis-based on principal curves and neural networks. Computers \& Chemical Engineering 1996, 20, 65-78.

40. McCalden, R. W.; McGeough, J. A. Age-related changes in the compressive strength of cancellous bone. The relative importance of changes in density and trabecular architecture. The Journal of Bone \& Joint Surgery 1997, 79, 421-427.

41. Gibson, L. J. Biomechanics of cellular solids. Journal of Biomechanics 2005, 38, 377-399.

42. Beale, M. Adapt network with weight and bias learning rules - MATLAB adaptwb. See http://www.mathworks.co.uk/help/ nnet/ref/adaptwb.html for further details. Accessed 09/03/2014.

43. Peteiro-Barral, D.; Guijarro-Berdiñas, B. A study on the scalability of artificial neural networks training algorithms using multiple-criteria decision-making methods. In Artificial Intelligence and Soft Computing (Rutkowski, L.; Korytkowski, M.; Scherer, R.; Tadeusiewicz, R.; Zadeh, L.; Zurada, J. (eds.)). Berlin: Springer Berlin Heidelberg, 2013, 162-173.

\section{WHAT DO YOU THINK?}

To discuss this paper, please email up to 500 words to the managing editor at bbn@icepublishing.com

Your contribution will be forwarded to the author(s) for a reply and, if considered appropriate by the editor-inchief, will be published as a discussion in a future issue of the journal.

ICE Science journals rely entirely on contributions sent in by professionals, academics and students coming from the field of materials science and engineering. Articles should be within 5000-7000 words long (short communications and opinion articles should be within 2000 words long), with adequate illustrations and references. To access our author guidelines and how to submit your paper, please refer to the journal website at www.icevirtuallibrary.com/bbn 